\title{
Creation and Implementation of an Emergency Trolley in the Pediatric Depart- ment at University Teaching Hospital of Butare (CHUB): The Way Forward for Quality Resuscitation
}

\author{
Charles Twagirayezu ${ }^{1}$, Stephanie Taylor ${ }^{1}$ \\ ${ }^{1}$ University Teaching Hospital of Butare, Rwanda
}

\section{Background}

In January 2014, the In-Charge Nurse of Pediatrics at CHUB observed that materials necessary for providing life saving measures were not readily available. With the collaboration of the HRH pediatric nurse an emergency trolley was developed and put into use in May 2014. The purpose of this project was to improve the availability, knowledge of, and use of emergency materials within the pediatric department at CHUB.

\section{Description}

The pediatric department at CHUB receives critically ill children into its 3-bed emergency room with an average of 6 children requiring resuscitation in the department a month. A pediatric emergency trolley was developed after a literature review, input from colleagues, and consulting CHUB's pharmacy. The emergency trolley was presented to physicians and nurses to highlight its importance prior to and post implementation. Topics reviewed included: the location of the trolley, location of equipment, demonstrations and explanations as to the purpose of it, and the process to report missing equipment.

\section{Lessons Learned}

Holding discussions with physicians and nurses were critical prior to implementation to ensure that all necessary materials for resuscitation were found in the trolley. This meant including both emergency and every day materials such as gloves because it is difficult to quickly obtain all equipment in emergency situations. Consulting pharmacy to discuss availability and appropriateness (for example, not including intubation medications because they require specific training) of materials was vital to developing a completely functioning emergency trolley. Maintaining the trolley continues to be a challenge; however, the development of a daily checklist and daily nurse assignment has increased re-stocking of materials.

\section{Conclusions}

Many variables were considered when creating and implementing the emergency trolley and it has had a positive effect on resuscitation practices in the pediatric department at CHUB. Healthcare personnel reported they were more confident in resuscitations, were aware of the purpose of using an emergency trolley, and it has served as a model for the neonatal unit. Currently, research is being conducted to create and improve the emergency trolleys in other departments within CHUB.

Key words: emergency trolley, resuscitation, pediatrics, limited resource setting 